\title{
Crescimento de mudas de pau-brasil (Caesalpinia echinata Lam.), submetidas a cinco níveis de sombreamento
}

\author{
Francismar Francisco Alves Aguiar ${ }^{1}$, Shoey Kanashiro ${ }^{2}$, Armando Reis Tavares ${ }^{2 *}$, \\ Thais Denise Rodrigues do Nascimento ${ }^{3}$,Fabrício Melito Rocco ${ }^{3}$
}

\begin{abstract}
RESUMO
O objetivo deste estudo foi avaliar o crescimento de mudas de pau-brasil (Caesalpinia echinata Lam.), submetidas a cinco níveis de sombreamento. As mudas, com 18 meses de idade, foram submetidas aos níveis de 0, 20, 40, 60 e 80\% de sombreamento, sob estrutura de telados pretos, tipo sombrite. Os maiores valores para altura foram obtidos em plantas cultivadas sob 20, 40 e $60 \%$ de sombreamento. Plantas sob pleno sol e sob $20 \%$ de sombreamento apresentaram maiores diâmetros do colo e menores relações entre altura e diâmetro do colo, o que revela maior equilíbrio no crescimento. O maior número de folhas foi obtido em plantas sob pleno sol. O aumento da luminosidade ocasionou maiores massas de matéria seca da parte aérea, do sistema radicular e total, além de menores índices entre massa de matéria seca da parte aérea e das raízes, o que sugere maior investimento de biomassa para as raízes. Os maiores valores do Índice de Qualidade de Dickson (IQD) foram observados em mudas cultivadas sob menores índices de sombreamento, sendo um bom indicador da qualidade das mudas.
\end{abstract}

Palavras-chave: Arbórea nativa, crescimento, mudas, sombreamento.

\section{ABSTRACT \\ Seedling development of a Brazilian wood species (Caesalpinia echinata Lam.) submitted to five levels of shading}

The aim of this study was to evaluate the growth of seedlings of Caesalpinia echinata Lam, subjected to five levels of shade. The seedlings, with 18 months of age, were subjected to 0\%, 20, 40, 60 and $80 \%$ shade, obtained by with black polypropylene screen. The highest values for heights were obtained in plants grown under 20, 40 and $60 \%$ shade. Plants in full sun and $20 \%$ shade showed a higher stem base diameter; lower height/stem base diameter ratio, which reveals more balanced growth. The larger number of leaves was obtained in plants under a full sun. Considering the biomass allocation, increasing luminosity occasioned higher shoot, root and total dry mass accumulation and lower shoot/root ratios, suggesting greather investment of biomass to the roots. The highest values of Dickson's Quality Index were observed in seedlings grown under lower levels of shading, being a good indicator of quality seedlings.

Key words: Native tree species, growth, seedlings, shading.

Received 22/12/2010 and accepted for publication 27/10/2011

${ }^{1}$ Engenheiro-Agrônomo, Mestre. Instituto de Botânica, Av. Miguel Stéfano, 3687, 04301-902, São Paulo, São Paulo, Brasil. francismaraguiar2000@yahoo.com.br ${ }^{2}$ Engenheiros-Agrônomos, Doutores. Instituto de Botânica, Av. Miguel Stéfano, 3687, 04301-902 São Paulo, São Paulo, Brasil. skanashi@uol.com.br; atavares2005@yahoo.com.br (autor para correspondência)

${ }^{3}$ Biólogos. Instituto de Botânica, Av. Miguel Stéfano, 3687, 04301-902 São Paulo, São Paulo, Brasil. 


\section{INTRODUÇÃO}

Os estudos sobre espécies ameaçadas de extinção, em especial aquelas ocorrentes em área de alto risco ambiental, são prioritários para definir estratégias de conservação (Lima, 2003). O pau-brasil (Caesalpinia echinata Lam.), ou ibirapitanga, pertencente à família Leguminosae e subfamília Caesalpinioideae, é espécie nativa do Brasil, de ocorrência natural desde o Rio Grande do Norte até o Rio de Janeiro, na floresta pluvial Atlântica (Lamarca et al., 2009).

Estudos sobre a adaptação das espécies arbóreas à disponibilidade de luz no seu ambiente de crescimento são importantes, no sentido de contribuir para o desenvolvimento de técnicas de plantio e de manejo de mudas dessas espécies, na perspectiva de múltiplos usos da floresta (Lima et al., 2010). Os viveiros produtores de mudas de espécies florestais têm como principal problema determinar, durante a fase de viveiro, quais fatores alteram a sobrevivência e o desenvolvimento inicial das mudas no campo. Os atributos das mudas, necessários para obtenção do sucesso do plantio no campo, são denominados "qualidade de muda" (Fonseca et al., 2002).

Estudos de crescimento de plantas, por meio do controle da radiação incidente por uso de telados redutores da radiação (Aguilera et al., 2004), têm sido amplamente utilizados para o conhecimento da ecofisiologia de espécies, submetidas a diferentes condições de luminosidade. Os parâmetros morfológicos são os mais utilizados na determinação do padrão de qualidade das mudas, em vista de sua compreensão mais intuitiva, por parte dos viveiristas, enquanto os parâmetros fisiológicos são de difícil mensuração e análise (Chaves \& Paiva, 2004).

Estudos sobre o crescimento de árvores nativas têm sido feitos para o conhecimento da resposta de diversas espécies submetidas a diferentes condições de luminosidade, tendo sido observado que a eficiência no crescimento da planta pode ser relacionada com a habilidade de adaptação de plântulas às condições luminosas do ambiente (Silva et al., 2007). A variabilidade entre as diferentes espécies, quanto à necessidade de luz, durante as etapas do ciclo vital e hábitos de crescimento, resultam no desenvolvimento de comunidades estratificadas, que proporcionam ampla faixa de habitats, importante na adaptação das espécies à luminosidade ambiental, principalmente na fase juvenil, por condicionar mudanças morfogenéticas e fisiológicas na sua estrutura e função (Whatley \& Whatley, 1982).

Lorenzi (1992) caracterizou a espécie C. echinata como heliófita ou esciófita. Aguiar \& Barbedo (1996), entretanto, a classificaram como semi-heliófita, recomendando sombreamento moderado na fase juvenil. Aguiar et al. (2005) obtiveram maior crescimento em altura e diâmetro, em mudas de C. echinata com 12 meses de idade, sob tela com
$60 \%$ de sombreamento e Aguiar \& Barbedo (1996), combinando três níveis de luminosidade e dois de temperatura, observaram o aumento na velocidade de crescimento (altura do caule) quando o sombreamento foi de até 50\%.

O objetivo deste estudo foi avaliar o crescimento e a qualidade de mudas de C. echinata submetidas a diferentes níveis de sombreamento.

\section{MATERIAL E MÉTODOS}

O presente trabalho foi realizado no Instituto de Botânica, São Paulo, Estado de São Paulo, nos meses de dezembro de 2000 a maio de 2003. As sementes de C. echinata foram obtidas de frutos coletados no Arboreto Experimental de Pau-brasil, em Moji-Guaçu, SP. Após o beneficiamento, as sementes foram semeadas em tubetes, contendo o substrato comercial à base de casca de Pinus, turfa, vermiculita expandida e carvão moído (recomendado para café), sob telado com 50\% de sombreamento.

Aos cinco meses de idade, as mudas foram transplantadas para sacos plásticos pretos, de $20 \mathrm{~cm}$ de altura por $15 \mathrm{~cm}$ de diâmetro, preenchidos com o substrato comercial (Plantmax-café ${ }^{\circledR}$ ) e aos 18 meses foram transplantadas para sacos plásticos de $40 \mathrm{~cm}$ de altura por $20 \mathrm{~cm}$ de diâmetro, com o mesmo substrato.

As mudas foram submetidas a cinco níveis de sombreamento: $0,20,40,60$ e $80 \%(2.420,1.760,1.060,666$ e 412 $\mu \mathrm{mol} \mathrm{m} \mathrm{m}^{2} \mathrm{~s}^{-1}$ respectivamente), sob estrutura de telados pretos de polietileno (tipo sombrite), com dimensões $2,0 \mathrm{x}$ 2,0 x 2,0 m. A manutenção do experimento foi feita com irrigação por aspersão, duas vezes por semana, e adubação de base com $15 \mathrm{~g}$ por planta do fertilizante osmocote (15:10:10).

As variáveis altura da muda $(\mathrm{H})$, diâmetro à altura do colo (D), relação entre altura e diâmetro da muda (H/D) e número de folhas foram avaliadas a cada 90 dias, durante o período de 720 dias. Ao final do experimento, avaliaramse, também, as variáveis massa de matéria seca da parte aérea (PMSA), radicular (PMSR) e total da planta (PMST), relação entre PMSA e PMSR e o índice de qualidade de Dickson (IQD) (Chaves \& Paiva, 2004).

O delineamento experimental adotado foi em blocos casualizados, com quatro blocos e cinco tratamentos, totalizando 20 parcelas, com oito plantas cada. As médias obtidas para as variáveis analisadas a cada 90 dias foram submetidas à análise de variância da regressão e as médias obtidas ao final do experimento (720 dias), comparadas pelo teste de Tukey, a 5\% de probabilidade.

\section{RESULTADOS E DISCUSSÃO}

As mudas de C. echinata apresentaram comportamento semelhante em relação à altura, nos tratamentos $0,20 \mathrm{e}$ $40 \%$ de sombreamento, desde o início do experimento, 
evidenciado pela proximidade entre as linhas de tendência (Figura 1). O maior valor para a altura da muda, ao final do experimento (Tabela 1), foi obtido em plantas cultivadas sob telas com $40 \%$ de sombreamento; entretanto, não significativo, quando comparado com os dos tratamentos 0,20 e $60 \%$ de sombreamento, sendo que as mudas no tratamento $80 \%$ de sombreamento apresentaram o menor valor para esta variável. Em ambientes sombreados algumas espécies apresentam rápido crescimento como mecanismo de adaptação, visando a escapar ao déficit de luz, já que elas não são capazes de tolerar baixas intensidades luminosas, por meio do reajuste de suas taxas metabólicas (Morais Neto et al., 2000). Aguiar \& Barbedo (1996) mostraram o aumento na velocidade de crescimento em altura de $C$. echinata, quando sombreada a $50 \%$ de luminosidade; entretanto, mudas desenvolvidas sob luminosidade total e sob $80 \%$ de sombreamento apresentaram valores próximos em altura, diferentes dos resultados obtidos no presente estudo. As diferenças entre espécies adaptadas ao sol ou à sombra não são claras, em florestas tropicais; indivíduos podem germinar em um ambiente e se desenvolver em outro, ou mesmo em ambientes contrastantes e que se alternam, antes de atingir o dossel. Segundo Mengarda et al. (2009), C. echinata seria uma espécie intermediária entre plantas tolerantes a altas intensidades luminosas e plantas com tolerância a ambientes sombreados, ocorrendo dentro de uma sucessão ecológica entre as espécies pioneiras e as espécies clímax.

O diâmetro do colo das plantas (Figura 2) apresentou curvas de crescimento semelhantes, em todos os tratamentos; entretanto, a redução da luminosidade ocasionou diminuição do diâmetro do colo. Ressalta-se que as linhas de tendência dos tratamentos com maior luminosidade ( 0 e $20 \%$ de sombreamento) permaneceram praticamente sobrepostas durante o período. Plantas cultivadas sob pouco $(20 \%)$ ou nenhum $(0 \%)$ sombreamento apresentaram os maiores valores e diferiram das mantidas sob 60 e $80 \%$ de sombreamento, o menor valor para a variável diâmetro (Tabela 1). O crescimento do diâmetro do caule depende da atividade cambial que, por sua vez, é estimulada por carboidratos produzidos pela fotossíntese e por hormônios translocados das regiões apicais; é, portanto, um bom indicador da assimilação líquida (Paiva et al., 2003), indicando que a espécie $C$. echinata apresenta comportamento heliófilo. Resultados semelhantes foram obti-

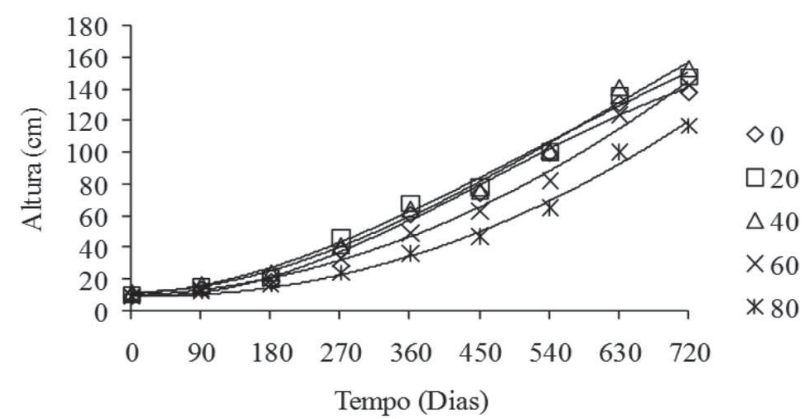

Equações de regressão e coeficientes de determinação: $0 \%: \mathrm{y}=-5 \mathrm{E}-07 \mathrm{x} 3+0,0006 \mathrm{x} 2-0,0389 \mathrm{x}+10,759\left(\mathrm{R}^{2}=0,9934 * *\right)$ $20 \%: \mathrm{y}=-3 \mathrm{E}-07 \mathrm{x}^{3}+0,0004 \mathrm{x}^{2}+0,0211 \mathrm{x}+10,568\left(\mathrm{R}^{2}=0,9901 * *\right)$ $40 \%: \mathrm{y}=-2 \mathrm{E}-07 \mathrm{x}^{3}+0,0004 \mathrm{x}^{2}+0,0017 \mathrm{x}+11,896\left(\mathrm{R}^{2}=0,9913^{* *}\right)$ $60 \%: y=0,0002 x^{2}+0,0182 x+10,543\left(R^{2}=0,9924 * *\right)$ $80 \%: y=0,0002 x^{2}-0,016 x+10,745\left(R^{2}=0,9912 * *\right)$

Figura 1. Altura de mudas de pau-brasil (Caesalpinia echinata Lam.), submetidas a diferentes níveis de sombreamento $(0,20$, $40,60$ e $80 \%)$.

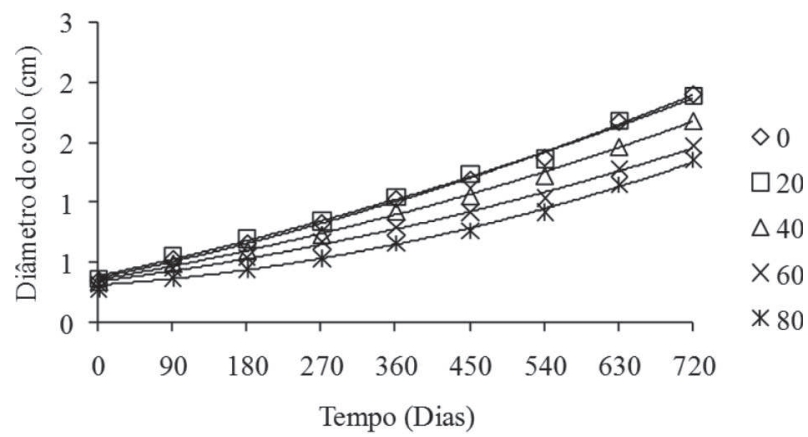

Equações de regressão e coeficientes de determinação: $0 \%: \mathrm{y}=1 \mathrm{E}-06 \mathrm{x}^{2}+0,0014 \mathrm{x}+0,3711\left(\mathrm{R}^{2}=0,998 * *\right)$ $20 \%: \mathrm{y}=8 \mathrm{E}-07 \mathrm{x}^{2}+0,0015 \mathrm{x}+0,3934\left(\mathrm{R}^{2}=0,9964 * *\right)$ $40 \%: \mathrm{y}=9 \mathrm{E}-07 \mathrm{x}^{2}+0,0012 \mathrm{x}+0,3662\left(\mathrm{R}^{2}=0,9981 * *\right)$ $60 \%: \mathrm{y}=9 \mathrm{E}-07 \mathrm{x}^{2}+0,0009 \mathrm{x}+0,344\left(\mathrm{R}^{2}=0,9958 * *\right)$ $80 \%: y=1 E-06 x^{2}+0,0005 x+0,3119\left(R^{2}=0,997 * *\right)$

Figura 2. Diâmetro do colo de mudas de pau-brasil (Caesalpinia echinata Lam.), submetidas a diferentes níveis de sombreamento $(0,20,40,60$ e $80 \%)$.

Tabela 1. Médias da altura, diâmetro do colo, número de folhas e relação entre altura e diâmetro do colo (H/D) de mudas de Caesalpinia echinata (pau-brasil) aos 720 dias após repicagem

\begin{tabular}{lcccc}
\hline Sombreamento (\%) & Altura $(\mathbf{c m})$ & Diâmetro $(\mathbf{c m})$ & $\mathbf{N}^{\mathbf{0}}$ de folhas & H/D \\
\hline 0 & $137,84 \mathrm{ab}$ & $1,91 \mathrm{a}$ & $41,2 \mathrm{a}$ & $72,38 \mathrm{c}$ \\
20 & $148,11 \mathrm{a}$ & $1,88 \mathrm{a}$ & $29,0 \mathrm{~b}$ & $78,81 \mathrm{bc}$ \\
40 & $152,98 \mathrm{a}$ & $1,69 \mathrm{ab}$ & $27,5 \mathrm{~b}$ & $90,76 \mathrm{ab}$ \\
60 & $142,45 \mathrm{a}$ & $1,48 \mathrm{bc}$ & $23,1 \mathrm{~b} \mathrm{c}$ & $96,64 \mathrm{a}$ \\
80 & $117,27 \mathrm{~b}$ & $1,35 \mathrm{c}$ & $19,2 \mathrm{c}$ & $86,46 \mathrm{ab}$ \\
\hline $\mathrm{CV}(\%)$ & 7,25 & 6,20 & 12,80 & 6,52 \\
\hline
\end{tabular}

Médias seguidas da mesma letra na coluna não diferem, significativamente, entre si $(\alpha<0,05)$ pelo teste Tukey. 
dos em Ochroma lagopus Sw. (pau-de-balsa), em que o diâmetro do colo foi favorecido pela alta luminosidade (Campos \& Uchida, 2002).

A relação H/D (Figura 3) mostrou tendência de diminuição da relação entre altura e o diâmetro do colo, em plantas cultivadas sob até $40 \%$ de sombreamento, o que reflete maior equilíbrio no desenvolvimento dessas mudas. Nas plantas que permaneceram sob maior sombreamento (60 e 80\%), a relação H/D tendeu a aumentar, mostrando maior crescimento em altura em detrimento do diâmetro do colo. Os maiores valores da relação H/D foram obtidos em mudas cultivadas a partir de $40 \%$ de sombreamento, sendo que as cultivadas sob $60 \%$ de sombreamento apresentaram o maior desequilíbrio no desenvolvimento simultâneo de altura da planta e diâmetro do colo, e, as cultivadas sem sombreamento, o menor valor H/D (Tabela 1). A relação entre esses dois parâmetros é uma variável que indica a qualidade de mudas a serem levadas ao campo, uma vez que se espera equilíbrio no seu desenvolvimento e o menor valor da relação H/D implica mudas mais resistentes no campo, como observado em mudas de jatobá (Hymenaea courbaril L.) (Campos \& Uchida, 2002).

O aumento do número de folhas mostrou comportamento similar para os diferentes níveis de sombreamento, evidenciado pela proximidade entre as curvas de crescimento (Figura 4). Este fato foi observado até cerca de 500 dias após o início do cultivo, momento em que houve grande aumento no número de folhas nas mudas mantidas sob luz plena ( $0 \%$ de sombreamento). A maior quantidade de folhas foi obtida em mudas mantidas sob pleno sol, enquanto os níveis de 60 a $80 \%$ de sombreamento apresentaram os menores valores médios (Tabela 1). Visualmente, observou-se maior expansão das folhas do pau-

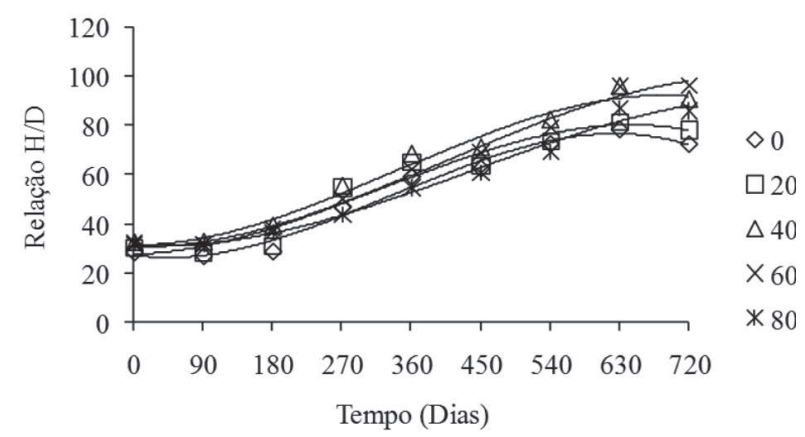

Equações de regressão e coeficientes de determinação: $0 \%: \mathrm{y}=-5 \mathrm{E}-07 \mathrm{x}^{3}+0,0005 \mathrm{x}^{2}-0,0455 \mathrm{x}+27,417\left(\mathrm{R}^{2}=0,9802 * *\right)$ $20 \%: \mathrm{y}=-4 \mathrm{E}-07 \mathrm{x}^{3}+0,0004 \mathrm{x}^{2}+0,0046 \mathrm{x}+28,058\left(\mathrm{R}^{2}=0,9487 * *\right)$ $40 \%: \mathrm{y}=-4 \mathrm{E}-07 \mathrm{x}^{3}+0,0004 \mathrm{x}^{2}+4 \mathrm{E}-05 \mathrm{x}+31,617\left(\mathrm{R}^{2}=0,9805 * *\right)$ $60 \%: y=-3 E-07 x^{3}+0,0003 x^{2}-0,0072 x+31,087\left(R^{2}=0,9903 * *\right)$ $80 \%: y=-2 E-07 x^{3}+0,0003 x^{2}-0,0273 x+32,367\left(R^{2}=0,9862 * *\right)$

Figura 3. Relação entre altura e diâmetro do colo de mudas de paubrasil (Caesalpinia echinata Lam.), submetidas a diferentes níveis de sombreamento $(0,20,40,60$ e $80 \%)$. brasil nos locais sombreados, quando comparada com a das folhas das plantas cultivadas sob pleno sol, as quais se apresentaram menores e mais espessas (não avaliado), enquanto as das plantas cultivadas em condições de sombreamento eram mais delgadas e menos ramificadas do que as crescidas sob pleno sol, indicando que existe plasticidade nas folhas da espécie, adaptando-se às diferentes condições de sombreamento. Resultados semelhantes foram obtidos por Silva et al. (2007) na espécie Hymenaea parvifolia Huber (jutaí-mirim), a qual foi capaz de se ajustar para maximizar a aquisição de luz, mesmo em condição muito limitante, como a proporcionada pelo sombreamento natural. Segundo Souza et al. (2007), mudas de C. echinata, cultivadas sob pleno sol, apresentaram injúrias, como queimaduras nas bordas dos folíolos; entretanto, o número de folhas foi equivalente ao das plantas sombreadas. Segundo os autores, o melhor desempenho foi verificado com plantas sob 30 e $70 \%$ de sombreamento, que apresentaram maior número de folíolos e diâmetro do caule mais espesso.

As maiores produções de fitomassa foram observadas nas plantas submetidas às condições de alta luminosidade (Tabela 2), comportamento também observado por Fanti \& Perez (2003), em mudas de Adenanthera pavonina L. (olho-de-dragão). O maior valor médio para a massa de matéria seca da parte aérea foi obtido em plantas cultivadas sem sombreamento, devendo estar relacionado com a maior quantidade de folhas, conforme mostrado na Tabela 1 e, portanto, com maior atividade fotossintética, fixação de carbono e aumento de massa. A redução da luminosidade ocasionou diminuição da biomassa da parte aérea.

A plena luminosidade provocou o aumento da massa da matéria seca do sistema radicular; resultados seme-

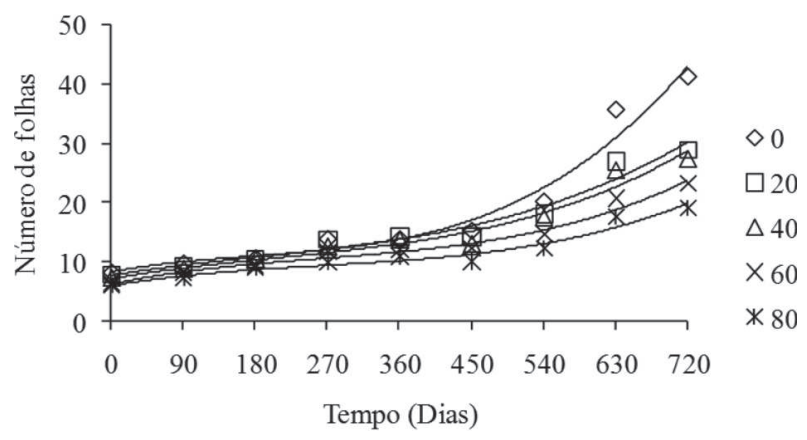

Equações de regressão e coeficientes de determinação: $0 \%: \mathrm{y}=2 \mathrm{E}-07 \mathrm{x}^{3}-9 \mathrm{E}-05 \mathrm{x}^{2}+0,0256 \mathrm{x}+8,328\left(\mathrm{R}^{2}=0,9659 * *\right)$ $20 \%: \mathrm{y}=8 \mathrm{E}-08 \mathrm{x}^{3}-4 \mathrm{E}-05 \mathrm{x}^{2}+0,022 \mathrm{x}+7,8897\left(\mathrm{R}^{2}=0,9571 * *\right)$ $40 \%: \mathrm{y}=8 \mathrm{E}-08 \mathrm{x}^{3}-5 \mathrm{E}-05 \mathrm{x}^{2}+0,0238 \mathrm{x}+7,3283\left(\mathrm{R}^{2}=0,9584 * *\right)$ $60 \%: \mathrm{y}=9 \mathrm{E}-08 \mathrm{x}^{3}-7 \mathrm{E}-05 \mathrm{x}^{2}+0,0284 \mathrm{x}+6,3606\left(\mathrm{R}^{2}=0,9729 * *\right)$ $80 \%: y=7 \mathrm{E}-08 \mathrm{x}^{3}-6 \mathrm{E}-05 \mathrm{x}^{2}+0,0232 \mathrm{x}+6,1448\left(\mathrm{R}^{2}=0,9531 * *\right)$

Figura 4. Número de folhas de mudas de pau-brasil (Caesalpinia echinata Lam.), submetidas a diferentes níveis de sombreamento $(0,20,40,60$ e $80 \%)$. 
Tabela 2. Médias de massa de matéria seca da parte aérea (PMSA), raízes (PMSR) e total (PMST), relação PMSA/PMSR e índice de qualidade de Dickson (IQD) de mudas de Caesalpinia echinata (pau-brasil) aos 720 dias após repicagem

\begin{tabular}{|c|c|c|c|c|c|}
\hline Sombreamento (\%) & PMSA (g) & $\operatorname{PMSR}(\mathrm{g})$ & PMST (g) & PMSA/PMSR & IQD \\
\hline 0 & $158,56 \mathrm{a}$ & $93,27 \mathrm{a}$ & $251,82 \mathrm{a}$ & $1,99 \mathrm{~b}$ & $3,46 \mathrm{a}$ \\
\hline 20 & $140,23 \mathrm{ab}$ & $75,62 \mathrm{ab}$ & $215,84 \mathrm{a}$ & $1,96 \mathrm{~b}$ & $2,68 a b$ \\
\hline 40 & $135,39 \mathrm{ab}$ & $60,11 \mathrm{abc}$ & $195,50 \mathrm{ab}$ & $2,31 \mathrm{ab}$ & $2,10 \mathrm{bc}$ \\
\hline 60 & $99,13 \mathrm{bc}$ & 33,37 bc & $132,50 \mathrm{bc}$ & $3,00 \mathrm{ab}$ & $1,33 \quad \mathrm{c}$ \\
\hline 80 & $67,24 \quad c$ & $23,03 \quad \mathrm{c}$ & $90,27 \quad c$ & $3,33 \mathrm{a}$ & 1,00 \\
\hline $\mathrm{CV}(\%)$ & 16,28 & 35,63 & 12,80 & 6,52 & \\
\hline
\end{tabular}

Médias seguidas da mesma letra na coluna não diferem, significativamente, entre si $(\alpha<0,05)$ pelo teste Tukey.

lhantes foram observados por Campos \& Uchida (2002), quando o sombreamento de $80 \%$ também provocou menor massa de matéria seca das raízes, em mudas de jatobá (Hymenaea courbaril). Segundo os autores, o aumento no crescimento do sistema radicular ocorreu em função da necessidade das mudas de absorverem maiores quantidades de água, por causa da maior transpiração e ressecamento do solo, quando cultivadas sob pleno sol, observação corroborada pela melhor relação PMSA/PMSR e pelo alto número de mudas mortas sob pleno sol, durante o experimento. A relação PMSA/PMSR diminuiu com o aumento da luminosidade (Tabela 2), mostrando maior equilíbrio na produção de matéria seca entre os sistemas aéreo e radicular, conforme também observado por Campos \& Uchida (2002). Resultados semelhantes foram encontrados por Duz et al. (2004) para Cedrela fissilis Vell. (cedro) e Cecropia glaziovi Snethl. (embaúba), cultivadas em diferentes regimes de luz.

As mudas com maior IQD foram as cultivadas sob $0 \mathrm{e}$ $20 \%$ de sombreamento, mostrando a melhor qualidade das mudas sob menor sombreamento (Tabela 2). O IQD é um bom indicador da qualidade das mudas, pois no seu cálculo são considerados a robustez e o equilíbrio da distribuição da biomassa na muda, ponderando-se os resultados de vários parâmetros importantes, empregados para a avaliação da qualidade (Fonseca et al., 2002). Hunt (1990) adotou o valor de 0,20 como o mínimo para o IQD, para avaliação de mudas de Pinus; segundo este parâmetro, todas as mudas de $C$. echinata apresentaram qualidade para serem usadas para plantio.

\section{CONCLUSÕES}

Caesalpinia echinata apresentou características de plantas heliófilas, mostrando maior crescimento e mudas com melhor relação do sistema radicular e aéreo, assim como para o IQD, sob condições de maior luminosidade.

\section{REFERÊNCIAS}

Aguiar FFA \& Barbedo CJ (1996) Efeito de fatores ambientais no crescimento de mudas de pau-brasil (Caesalpinia echinata Lam.). Revista Brasileira de Horticultura Ornamental, 2:26-32.
Aguiar FFA, Kanashiro S, Tavares AR, Mecca Pinto M, Stancato GC, Aguiar J \& Nascimento TDR (2005) Germinação de sementes e formação de mudas de Caesalpinia echinata Lam. (pau-brasil): efeito de sombreamento. Revista Árvore, 29:871875 .

Aguilera DB, Ferreira FA \& Cecon PR (2004) Crescimento de Siegesbeckia orientalis sob diferentes condições de luminosidade. Planta Daninha, 22:43-51.

Campos MAA \& Uchida T (2002) Influência do sombreamento no crescimento de mudas de três espécies amazônicas. Pesquisa Agropecuária Brasileira, 37:281-288.

Chaves AS \& Paiva HN (2004) Influência de diferentes períodos de sombreamento sobre a qualidade de mudas de fedegoso (Senna macranthera (Collad.) Irwin et Barn.). Scientia Forestalis, 65:2229.

Duz SR, Siminski A, Santos M \& Paulilo MTS (2004) Crescimento inicial de três espécies arbóreas da Floresta Atlântica em resposta à variação na quantidade de luz. Revista Brasileira de Botânica, 27:587-596.

Fanti SC \& Perez SCJGA (2003) Influência do sombreamento artificial e da adubação química na produção de mudas de Adenanthera pavonina L. Ciência Florestal, 13:49-56.

Fonseca EP, Valéri SA, Miglioranza E, Fonseca NAN \& Couto L (2002) Padrão de qualidade de mudas de Trema micrantha (L.) Blume, produzidas sob diferentes períodos de sombreamento. Revista Árvore, 26:515-523.

Hunt GA (1990) Effect of styroblock design and cooper treatment on morphology on conifer seedlings. In: Target seedlings symposium, meeting of the Western Forest nursery associations. Roseburg, USDA-Forest Service. p.218-222.

Lamarca EV, Leduc SNM \& Barbedo CJ (2009) Viabilidade e vigor de sementes de Caesalpinia echinata Lam. (pau-brasil Leguminosae) pelo teste de tetrazólio. Revista Brasileira de Botânica, 32:793-803.

Lima HC (2003) Taxonomia e distribuição geográfica atual de Caesalpinia echinata Lam. In: Simpósio "Pau-brasil: Ciência e Arte". São Paulo, FAPESP. CD ROM.

Lima MAO, Mielke MS, Lavinsky AO, França S, Almeida AAF \& Gomes FP (2010) Crescimento e plasticidade fenotípica de três espécies arbóreas com uso potencial em sistemas agroflorestais. Scientia Forestalis, 38:527-534.

Lorenzi H (1992) Árvores brasileiras - Manual de identificação e cultivo de plantas arbóreas nativas do Brasil. Nova Odessa, Editora Plantarum. 382p.

Mengarda LHG, Souza RLF, Campostrini E, Reis FO, Vendrame WA \& Cuzzuol GRF (2009) Light as an indicator of ecological succession in brazilwood (Caesalpinia echinata Lam.). Brazilian Journal of Plant Physiology, 21:55-64. 
Morais Neto SP, Gonçalves JLM, Takaki M, Cenci S \& Gonçalves JC (2000) Crescimento de mudas de algumas espécies arbóreas que ocorrem na mata atlântica em função do nível de luminosidade. Revista Árvore, 24:35-45.

Paiva LC, Guimarães RJ \& Souza CAS (2003) Influência de diferentes níveis de sombreamento sobre o crescimento de mudas de cafeeiro (Coffea arabica L.). Ciência e Agrotecnologia, 27:134-140.
Silva BM, Lima J, Dantas VA, Moraes W \& Zumkeller DS (2007) Efeito da luz no crescimento de mudas de Hymenaea parvifolia Huber. Revista Árvore, 31:1019-1026.

Souza RLF, Soneghet GP \& Cuzzuol GRF (2007) Crescimento inicial de Caesalpinia echinata Lam. em resposta à luminosidade. Revista Brasileira de Biociências, 5:978-980.

Whatley JM \& Whatley FR (1982) A luz e a vida das plantas. Temas de Biologia v.30. São Paulo, EDUSP. 101p. 